\title{
The effect of afferent stimuli on steadily discharging single motor units in man
}

\author{
IR WILLIAMS, M HAYWARD \\ From the Associated Unit of Neurological Science, University of Liverpool, and the Departments of \\ Neurology and Neurophysiology, Mersey Regional Department of Medical and Surgical Neurology, Walton \\ Hospital, Liverpool, UK
}

SUMMARY The effect of afferent stimuli on the firing pattern of single motor units has been studied in the human first dorsal interosseous muscle of the hand. A reproducible series of changes in the probability of motor unit firing was seen. There was evidence for an excitatory spinal reflex involving median nerve muscle afferent fibres and ulnar nerve motor fibres. Cutaneous and muscle afferent pathways expected to be important in the control of fine movement of the first digit produced an excitatory reflex response at about $70 \mathrm{~ms}$. All sites stimulated in the ipsilateral limb gave rise to a profound reduction in motor unit firing from about $70-130 \mathrm{~ms}$.

The control of fine movement is dependent upon afferent information. This is particularly true of skilled hand movement. It might, therefore, be expected that any sustained skilled movement would be altered by externally imposed changes in afferent activity. Studies on the silent period in the interference pattern of a contracting muscle, brought about by a stimulus to the skin or nerve supply, and of reflex responses in small hand muscles following cutaneous stimuli, have confirmed this. ${ }^{1-3}$ In the $150 \mathrm{~ms}$ after stimulation of nerve or skin during weak muscle contraction, a series of changes occurs in the pattern of firing of the anterior horn cell. Caccia et al, ${ }^{3}$ using surface electrodes and weak contractions of small hand muscles, demonstrated alternating periods of increased and decreased activity of motor units during this time. It was not clear from their study whether this reflected a series of changes in activity of individual anterior horn cells or whether different anterior horn cells behaved in different fashions giving rise to separate peaks of motor unit discharges in the muscle. Stephens and his colleagues ${ }^{4-6}$ have shown that within the first $70 \mathrm{~ms}$ after nerve or cutaneous stimulation, individual motor units do exhibit periods of increased and decreased firing. Ashby and Labelle ${ }^{7}$ made a detailed study of the synaptic connections to anterior horn cells supplying some lower limb muscles which also showed early changes in motor unit firing pattern.

Address for reprint requests: Dr IR Williams, Walton Hospital, Rice Lane, Liverpool L9 1 AE, UK.

Received 30 March 1981 and in revised form 13 July 1981 Accepted 25 August 1981
The changes in the firing pattern which take place more than 60 or $70 \mathrm{~ms}$ after a stimulus have not been studied in such detail.

It is evident from the work of Caccia et $a l^{3}$ that stimuli to a number of different sites can produce changes in the firing pattern of motor units within a muscle. They demonstrated that as the stimulus was moved across the hand, away from the muscle being studied, the reflex response became smaller. The most marked change which these authors described was a period of reduced activity around $90 \mathrm{~ms}$. More recent work, concentrating on the earlier changes, has not explored this further. The present study was undertaken to look at the prolonged period of reduced firing from 70-130 ms. It was necessary first to determine whether individual motor units did in fact exhibit the full series of changes described by Caccia et al. ${ }^{3}$ Thereafter the effects of carefully controlled electrical stimuli at different sites and at several intensities were investigated. In this way an attempt has been made to separate effects brought about through different pathways.

\section{Subjects and methods}

All studies were performed on healthy male volunteers aged 25-45 yr who were fully conversant with the nature of the procedures. A concentric needle electrode with a tip area of $0.019 \mathrm{~mm}^{2}$ was inserted into the first dorsal interosseous muscle. The subject was asked to activate a single motor unit and to maintain an approximately constant firing rate using the sound of the amplified unit and the oscilloscope display. Several single units could be studied in succession with only minor changes in the needle 


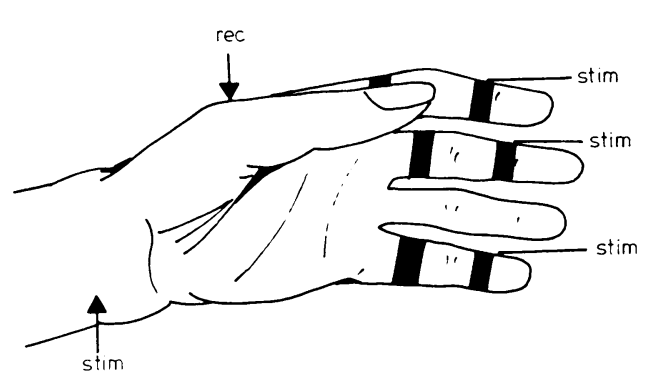

Fig 1 Position of recording and stimulating electrodes.

position. Ring electrodes around the proximal and middle phalanges were used to stimulate 2 nd, 3rd or 5 th digits. Bars $4 \mathrm{~cm}$ apart were used to stimulate the ulnar or median nerve at the wrist (fig 1). When the motor unit was firing steadily, electrical stimuli of $0 \cdot 1 \mathrm{~ms}$ duration were delivered from a constant current stimulator to one of the five sites. Stimulation was either regular at $1 \mathrm{~Hz}$ or pseudo-random with a mean frequency of $1.2 \mathrm{~Hz}$. During each study the stimulus current was constant but the effect of varying the current was examined at each site.

Orthodromic or antidromic sensory action potentials were recorded simultaneously with the motor unit recording using the electrodes described above. Motor unit discharges were amplified and displayed on a Medelec MS6 system and recorded on magnetic tape at two gains. A stimulus marker pulse was recorded on a 3rd channel (fig 2). During analysis of the results from the

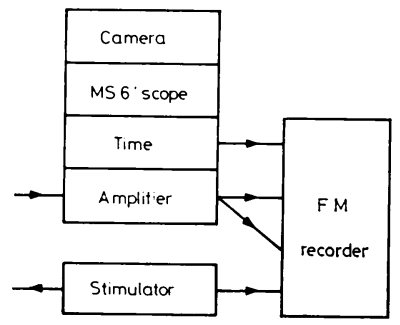

Fig 2 Diagram of arrangement for recording single motor unit potential and stimulus marker pulse.
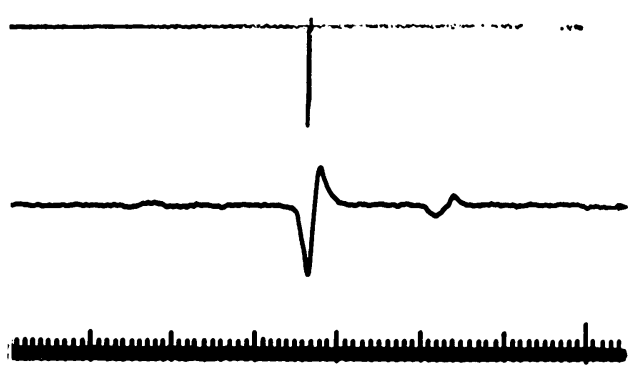

Fig 3 Single motor unit potential with its generated pulse above. Time scale shows 1 and 10 ms markers.

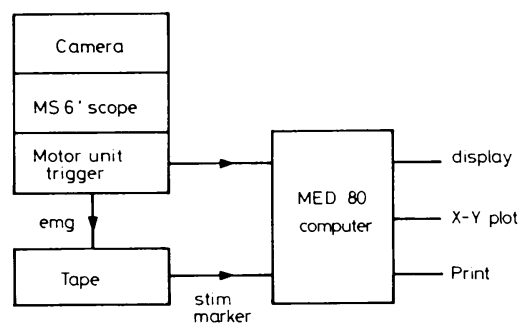

Fig 4 Diagram of arrangement for analysis of motor unit firing pattern after stimulus.

tape each motor unit potential was made to generate a marker pulse from a trigger circuit. The threshold of the trigger circuit was adjusted so as to exclude any other unit and to ensure that each unit discharge generated only one pulse (fig 3 ). This required continuous visual monitoring during each analysis and repeated analyses of each study. A photograph of each motor unit and its generated pulse was taken so that in subsequent calculations a correction could be made for the delay between the onset of the motor unit discharge and the generated pulse.

Each stimulus marker started a 512 ms period of motor unit counting in the MED 80 computer (Nicolet) with 256 bins each of 2 ms width (fig 4). The results of 174-900 (usually 300 ) such $512 \mathrm{~ms}$ periods were added together to give a histogram of motor unit activity following the stimulus. The histograms used in the figures do not use the trigger delay correction, but latencies quoted in the text are corrected for this small delay and are mean results for the several motor units studied. Latencies of periods of increase in activity are measured to peak because of the difficulty in ascertaining the onset. Periods of reduced motor unit activity are quoted as intervals containing no motor unit activity or in which only isolated discharges have been recorded. Each histogram displays the response of one motor unit only.

\section{Results}

STIMULATION OF IPSILATERAL ULNAR NERVE AT THE WRIST WITH STIMULI GREATER THAN MOTOR THRESHOLD

It was not possible to maintain the position of the intramuscular recording electrode when maximal contraction occurred in response to stimulation. The largest stimulus which did not prevent single unit recording was used. Five recordings were made from four motor units. The same pattern of responses was obtained in each study (fig 5a). The initial direct $M$ response was followed by a further peak at 28-30 ms. Between $M$ and 28-30 ms there was little activity. A third, rather broad, period of motor unit activity occurred at $60-70 \mathrm{~ms}$ and in two of the units studied there was a further peak at $180 \mathrm{~ms}$. From 80-130 ms there was little or no activity. 

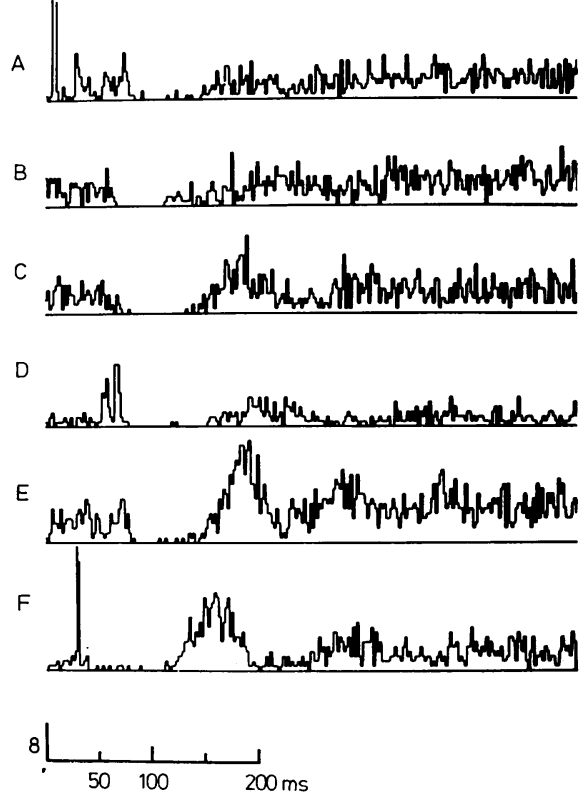

Fig 5 Histograms of the activity of a single motor unit in the first dorsal interosseous muscle after stimulation at (a) ulnar nerve at the wrist with stimuli above motor threshold, (255 stimuli). (b) ulnar nerve at the wrist with stimuli below motor threshold, (300 stimuli). (c) digit 5, (211 stimuli). (d) digit 2, (174 stimuli). (e) digit 3, (410 stimuli). ( $f)$ median nerve at the wrist, (301 stimuli). Vertical calibration is counts per 2 ms bin.

STIMULATION OF IPSILATERAL ULNAR NERVE AT THE WRIST, STIMULI BELOW MOTOR THRESHOLD

A stimulus was selected which gave a 10-20 $\mu \mathrm{V}$ antidromic sensory action potential at digit 5 but which elicited no motor unit activity in the 1st dorsal interosseous muscle. Twenty units were studied. Fifteen units showed a clear reduction in activity between 77-107 ms (fig 5b). Three others showed a reduction in firing for some part of this period. In the others there was no clear diminution of activity. Two units showed a clear peak at 170-180 ms and several others showed slightly increased firing probability. No consistent early changes were seen but two units had increased firing at 60-70 ms.

STIMULATION OF IPSILATERAL DIGIT 5

Thirteen units were studied, six at low stimulus intensity and 10 at high intensity. The lower stimulus was of sufficient strength to give a maximal ascending sensory action potential at the wrist, being approximately three times the threshold for sensory perception. Stimuli of this strength had little effect on the firing pattern of the motor unit. Small changes could be seen, however, and when the stimulus was increased to the maximum that the subject could tolerate these changes became much more pronounced (fig 5c). The trough in the histogram is apparent at $79-128 \mathrm{~ms}$ with a subsequent peak at $180 \mathrm{~ms}$.

STIMULATION OF IPSILATERAL DIGIT 2

Seven units were studied using stimuli (a) sufficient to give a maximal ascending sensory action potential at the wrist, (b) $50 \%$ above this level and (c) the maximum that the subject could tolerate. With low stimulus current there was no observable effect on the firing pattern of the motor unit. Increasing the stimulus current led to characteristic changes (fig 5d, 6a, 6b and 6c). It was, however, necessary to use stimuli well above those required to give a maximal sensory action potential at the wrist in order to produce these changes. There was a peak with a mean latency of $67 \mathrm{~ms}$. From $81-135 \mathrm{~ms}$ there was a profound reduction in firing. A variable peak occurs at $182 \mathrm{~ms}$. Just over half the units studied also showed an earlier period of reduced activity with a mean latency of $46 \mathrm{~ms}$.

STIMULATION OF IPSILATERAL DIGIT 3

Six units were studied. The response was similar to that seen when digit 2 was stimulated but a higher stimulus current was required to achieve the response (figures $6 \mathrm{~b} \& 5 \mathrm{e}$ ). The peak at $67 \mathrm{~ms}$ seen with stimulation of digit 2 was only occasionally marked.

STIMULATION OF IPSILATERAL MEDIAN NERVE AT THE WRIST

Nine recordings were made from seven motor units. Unit 45
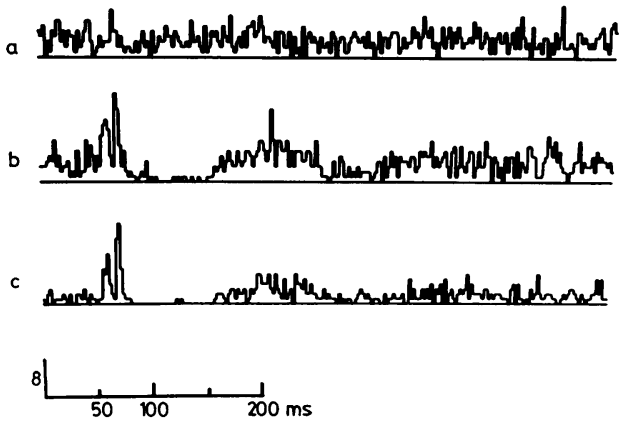

Fig 6 Histograms of the activity of a single motor unit in first dorsal interosseous muscle; effect of increasing stimulus current to digit 2: (a) at current giving maximal ascending sensory action potential at the wrist, (293 stimuli). (b) at current $50 \%$ above that used in a), (315 stimuli). (c) at maximum current tolerated by the subject, (174 stimuli). Vertical calibration is counts per $2 \mathrm{~ms}$ bin. 

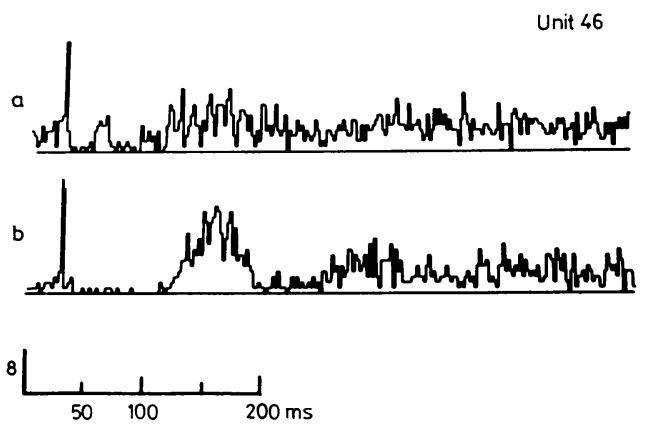

Fig 7 Histogram of the activity of a single motor unit in first dorsal interosseous muscle. Effect of increasing stimulus current to median nerve at wrist; (a) below motor threshold for the median nerve, (318 stimuli). (b) just above motor threshold for the median nerve, (301 stimuli). Vertical calibration is counts per 2 ms bin.

In order to reduce needle movement due to contraction of the thenar group of muscles and to prevent spread of the stimulus to the ulnar nerve, most studies were performed using stimuli below motor threshold for the median nerve. In three units the stimulus was then increased. The earliest change was a peak in firing activity at $31 \mathrm{~ms}$ after the stimulus (figs $5 \mathrm{f}$ and 7 ). Increasing the stimulus strength did not abolish this response. After depression of activity from $39-57$ ms there is a second peak at $61 \mathrm{~ms}$. This second peak diminishes as stimulus intensity is increased (fig 7). At all stimulus levels below maximal the mean period of depressed activity was from 70-105 ms but with maximum stimulation it began as early as $\mathbf{4 0} \mathrm{ms}$ after the stimulus. The late peak at 158 ms was of greater amplitude with higher current stimuli.

\section{STIMULATION AT OTHER SITES}

Stimulation of contralateral digits 2 or 5 at stimulus intensity up to the maximum tolerated had no effect on the firing pattern. Likewise stimulation of ulnar or median nerve at the opposite wrist with a current giving a maximum motor response did not alter the firing pattern of the nine motor units studied. Stimulation of the anterior tibial nerve at the ankle had no effect on the firing pattern of the three units studied in the first dorsal interosseous muscle of the hand.

\section{AUDITORY STIMULATION}

In one experiment, electrical stimuli to the nerve were replaced by 90 decibel clicks delivered to both ears through headphones at $1 \mathrm{~Hz}$. There was no effect on the firing pattern of the motor unit in the first dorsal interosseous muscle.
RELAXATION STUDIES

In order to test the ability to produce a silent period voluntarily, one subject was instructed to interrupt the regular firing of the single motor unit on feeling an electrical stimulus in the opposite hand. This proved to be a very difficult task. Motor unit activity continued well beyond $100 \mathrm{~ms}$, sometimes persisting for more than $150 \mathrm{~ms}$ after the stimulus. Following some stimuli motor unit activity continued unchanged in spite of the instruction.

\section{STIMULATION PATTERN}

At most sites the response to regular $1 \mathrm{~Hz}$ stimulation was compared with the response to pseudo-random stimuli with a mean frequency of $1 \cdot 2 \mathrm{~Hz}$. There was no major difference in the number or latency of peaks and troughs in the resulting histograms.

\section{Discussion}

These experiments are dependent upon the ability of the subject to fire an individual motor unit within a muscle at a relatively constant frequency. Control of the rate of firing of a motor unit by means of auditory and visual feedback is not part of normal motor control. The technique requires a much greater awareness of control on the part of the subject than is usual. Is it then possible that some of the effects described could be explained on the basis of interference with conscious control? In other words could a large electrical stimulus interrupt concentration allowing a decrease in motor unit activity? Three factors argue against this being an explanation for our results: Changes in motor unit activity that occur before about $50 \mathrm{~ms}$ have been initiated before the subject is conscious of the stimulus. In the experiments in which the subject was asked to cease voluntary firing of the motor unit as soon as possible after the stimulus was appreciated, it was rarely possible to do this before $150 \mathrm{~ms}$. The failure of auditory or other limb stimuli to effect a change in the firing pattern also suggests that the afferent stimuli did not produce their effect by interrupting concentration. It is not likely, therefore, that any of our results can be explained on the basis of a consciously determined response to the electrical stimulus.

A further aspect of the method should be considered. The stimulus was not synchronised with the motor unit discharge. When the unit was firing steadily at $10 \mathrm{~Hz}$ the interval between the stimulus and the previous discharge varied randomly between 0-100 ms. Following each stimulus motor unit activity was analysed for $512 \mathrm{~ms}$. When we analysed 200-300 such periods of $512 \mathrm{~ms}$ in which no stimulus was delivered an even distribution of motor unit 
activity was seen. The implications of this are important in understanding the significance of the results. The histogram does not represent a single sequence of events but reflects the probability of the motor unit firing at a given time after a stimulus. Because of the large number of trials summed in each histogram and the fact that the interval between the stimulus and the previous discharge was random, the changes of activity displayed in the histogram are independent of the time at which the unit last fired. Only when the stimulus has modified the pattern of discharges may periodicity lead to secondary peaks and depressions later in the histogram.

Stimulation of median or ulnar nerves, or of digits 2,3 or 5 , can alter the discharge pattern of the anterior horn cells innervating the first dorsal interosseous muscle of the hand. The series of changes in firing during the first $200 \mathrm{~ms}$ after stimulation is repeatable, constant and specific. The pattern and latency of the changes agree well with those described by Caccia et $a l^{3}$ and Garnett and Stephens. ${ }^{6}$ Whilst we studied only low threshold units which were recruited early, we found no evidence to suggest that sub-populations of motor units behave in fundamentally different ways. A few units did not respond to stimuli. It is not clear whether these are different types of unit, whether they have a higher threshold or whether technical difficulties interfered with the performance of the subject.

Changes in the first $30 \mathrm{~ms}$ after the stimulus are of limited interest. Only those stimuli to the ulnar nerve which were above motor threshold evoked an early direct motor response (M). Stimulation of sufficient strength to excite motor fibres gave rise to a second response between $25-30$ ms which was not present with stimuli below motor threshold. This was of lower amplitude in the histogram than the $\mathbf{M}$ response and is probably the $F$ response. ${ }^{8}$ This latency represents the shortest time for conduction to the spinal cord and back to the muscle. Changes in firing between $M$ and $25-30$ ms must be based on peripheral refractoriness and collision of impulses.

Stimulation of the median nerve at the wrist also produced a change in firing at about $25-30 \mathrm{~ms}$. This was sometimes present with low current and always present with high current stimulation. It was not seen when 2 nd or 3 rd digits were stimulated, even at high current. The latency indicates that it is a spinal reflex. The finding that it is only obvious following stimulation at the wrist, suggests either that muscle afferent nerves are involved or that more cutaneous afferent fibres can be excited by stimulation at the wrist than is possible by stimulating any single digit. The fact that the reflex appears before the motor threshold has been reached favours the conclusion that muscle afferent fibres are responsible. The implication of this observation is that afferent impulses from median innervated muscles can increase firing in an ulnar innervated muscle through a spinal reflex.

Those stimuli which produced an increase in firing probability at $30 \mathrm{~ms}$ also produced a later fall in firing probability. It appears that the period of inhibition is partly dependent upon the preceding excitation. With median nerve stimulation at the wrist the period of inhibition increases with the increase in firing at $30 \mathrm{~ms}$. This suggests that the inhibition is brought about at spinal level. Such spinal inhibition might be based on Renshaw cell activity. 239

A further period of increased probability of firing occurs between 50-70 ms when ulnar or median nerves are stimulated at the wrist and less frequently after stimulation of the 2 nd and 3 rd digits at high current. It does not occur with stimulation of digit 5 . This is the response discussed by Garnett and Stephens ${ }^{6}$ and is likely to be a "long loop" reflex. Cutaneous receptors in the 2 nd digit, as well as spindle afferents, must have great functional importance in the control of the first dorsal interroseous muscle which abducts that digit. There is good evidence that cutaneous and muscle afferent pathways project to the cortical motorneurones ${ }^{1011}$ and recordings from human motor cortex have suggested that an intact postcentral cortex is necessary for reflexes of this latency to occur. ${ }^{12}$

In our study, increasing median nerve stimulus current at the wrist and the accompanying increase in probability of firing at $30 \mathrm{~ms}$ led to a reduction in the response at $50-70 \mathrm{~ms}$ (fig 7). This suggests that the response at $50-70 \mathrm{~ms}$ is a suprasegmental reflex and that the resultant increase in descending activity in these pathways reaches the anterior horn cell during a period of spinal inhibition. The period of increased probability of firing between $50-70 \mathrm{~ms}$ was by far the most variable of the series of alterations in firing probability that we observed. The variability of this increase is also in keeping with the belief that it represents a suprasegmental "long loop" reflex.

Stimulation at every site studied in the ipsilateral limb was capable of producing a marked decrease in firing probability for a large part of the time between $70-130 \mathrm{~ms}$. It is interesting that stimulation of ulnar or median nerve at the wrist, with currents well below motor threshold, could produce absolute inhibition of firing during this period. In order to achieve a similar response when stimulating digits, it was necessary to use a much higher current than that needed to produce a maximum ascending sensory action potential. It is difficult to explain these two findings by a common mechanism. With small currents at the 
wrist only large, rapidly conducting fibres would have been excited. However, the increase in the effect when the stimulus to a digit was increased above that needed to give a maximal ascending sensory action potential, suggests that smaller fibres with a higher threshold can produce a similar effect. It is just possible that the high current used when stimulating the digit excited larger nerve fibres in nearby muscle or tendon, but this seems unlikely. Two or more entirely separate mechanisms must be responsible for the same inhibitory effect. When only the large diameter fibres of the ulnar nerve are stimulated at the wrist, the inhibition of motor unit firing after such a latency would appear to be determined at a suprasegmental level. An alternative explanation proposed by Caccia et $a l^{3}$ is that this period of decreased firing probability represents a reduction in spindle assistance. This assumes that following afferent stimulation there is an inhibition of gammamotorneurone discharge. This would in turn lead to a diminution of firing of $1 \mathrm{~A}$ afferents, thus removing some of the excitatory drive to the anterior horn cell. Whilst this hypothesis is conceivable for muscle afferent stimulation, the observation that a similar response can be obtained following cutaneous afferent stimulation makes it unlikely to be the only explanation.

In this study, the prolonged period of reduced firing from 70-130 ms was independent of any earlier change in firing. It did occur as the sole change. It is, therefore, not a secondary response dependent upon earlier firing of the unit and the subsequent periodicity inherent in the voluntary contraction. The origin of such powerful inhibition remains unknown.

Following the period of inhibition there is a variable increase in the firing probability with a peak at around $180 \mathrm{~ms}$. This is more prominent when high current stimuli are used and it is only present following a period of inhibition between $70-130 \mathrm{~ms}$. It, therefore, probably represents the commencement of the "normal" firing pattern after the prolonged period of inhibition. Although there is not a strict relationship between the degree of inhibition and the degree of increase in firing probability, it is in general true that with higher current stimuli the increase in firing probability around $180 \mathrm{~ms}$ is greater.
Events after $180 \mathrm{~ms}$ cannot be analysed with any ease. With an interspike interval varying around $100 \mathrm{~ms}$, periodicity begins to affect the pattern. When there is a large increase in firing at around $180 \mathrm{~ms}$ smaller peaks can be seen at approximately $100 \mathrm{~ms}$ thereafter.

\section{References}

${ }^{1}$ Merton PA. The silent period in a muscle of the human hand. J Physiol (Lond) 1951;114:183-98.

${ }^{2}$ Shahani BT, Young RR. Studies of the normal human silent period In: Desmedt JE, ed. New Developments in Electromyography and Clinical Neurophysiolog., vol 3. Basel: Karger, 1973:589-602.

${ }^{3}$ Caccia MR, McComas AJ, Upton ARM, Blogg T. Cutaneous reflexes in small muscles of the hand. $J$ Neurol Neurosurg Psychiatry 1973;36:960-77.

${ }^{4}$ Stephens JA, Usherwood TP, Garnett R. Technique for studying synaptic connections of single motorneurones in man. Nature 1976;263:343-4.

${ }^{5}$ Buller NP, Garnett R, Stephens JA. The reflex responses of single motor units in human hand muscles following muscle afferent stimulation. $J$ Physiol (Lond) 1980;303:337-49.

${ }^{6}$ Garnett R, Stephens JA. The reflex response of single motor units in human first dorsal interosseous muscle following cutaneous afferent stimulation. J Physiol (Lond) 1980;303:351-64.

'Ashby P, Labelle K. Effects of extensor and flexor group 1 afferent volleys on the excitability of individual soleus motorneurones in man. $J$ Neurol Neurosurg Psychiatry 1977;40:910-9.

${ }^{8}$ Mayer RF, Feldman RG. Observations on the nature of the F wave in man. Neurology (Minneap) 1967;17: 147-56.

${ }^{9}$ Renshaw B. Influence of discharge of motorneurones upon excitation of neighbouring motorneurones. $\boldsymbol{J}$ Neurophysiol $1941 ; 4: 167-83$.

${ }^{10}$ Lemon RN, Porter R. Afferent input to movementrelated precentral neurones in conscious monkeys. Proc Roy Soc B 1976;194:313-39.

${ }^{11}$ Goldring S, Ratcheson R. Human motor cortex; sensory input data from single neuron recordings. Science 1972;175:1493-5.

${ }^{12}$ Brown WF, Milner-Brown HS, Ball M, Girvin JP. Control of the motor cortex on spinal motor neurones in man. In: Desmedt JE, ed. Cerebral motor control in man: Long loop mechanisms. Progress in Clinical Neurophysiology, vol 4. Basel: Karger, 1978:246-62. 